\title{
Perspectives and Challenges in Molecular-Based Diagnostics and Personalized Treatment for Recurrent High-Grade Gliomas
}

\author{
Darius Kalasauskas ${ }^{1}$, Mirjam Renovanz ${ }^{1}$, Sven Bikar ${ }^{2}$, Anton Buzdin ${ }^{3,4,5}$, Ather Enam ${ }^{6}$, Sven Kantelhardt ${ }^{1}$, Alf Giese $^{6}$ and Ella L Kim $^{1^{*}}$ \\ ${ }^{1}$ Clinic for Neurosurgery, Johannes Gutenberg University Medical Centre, Mainz, Germany \\ ${ }^{2}$ Star SEQ GmbH, Mainz, Germany \\ ${ }^{3}$ National Research Centre Kurchatov Institute, Centre for Convergence of Nano, Bio, Information and Cognitive Sciences and Technologies, Moscow, Russia \\ ${ }^{4}$ First Oncology Research and Advisory Centre, Moscow, Russia \\ ${ }^{5}$ Rogachyov Federal Research Centre of Pediatric Hematology, Oncology and Immunology, Moscow, Russia \\ ${ }^{6}$ Department of Surgery, Section of Neurosurgery, Aga Khan University Hospital, Karachi, Pakistan
}

"Corresponding author: Ella L Kim, Clinic for Neurosurgery, Laboratory for Experimental Neurooncology, Johannes Gutenberg University Medical Centre, Langenbeckstrasse 1, 55131 Mainz, Germany, Tel: +496131178211; E-mail: ella.kim@unimedizin-mainz.de

Received date: March 27, 2017; Accepted date: April 18, 2017; Published date: April 24, 2017

Copyright: @ $2017 \mathrm{Kim}$ EL, et al. This is an open-access article distributed under the terms of the Creative Commons Attribution License, which permits unrestricted use, distribution, and reproduction in any medium, provided the original author and source are credited.

\begin{abstract}
Glioblastoma is the most common and most malignant type of intrinsic brain tumor in adults. The standard of care for glioblastoma consists of surgical debulking followed by combined radiochemotherapy. The clinical efficacy of standard therapies for newly diagnosed glioblastomas is rather modest with the highest survival rate at 5-years being less than $10 \%$. Inevitable recurrence after cytotoxic therapies poses the major challenge in the clinical management of high grade gliomas. For recurrent glioblastomas, there is no standard therapy with lack of level one evidence for treatment efficacy. Recent evidence indicates that post-therapy recurrence in gliomas is a consequence of a plethora of molecular and cellular factors including intratumoural heterogeneity, functional hierarchy of distinct types of glioma cells, dynamic changes in the molecular landscapes and cellular composition of the tumour during therapy and the impact of particular treatment modalities. There is an emerging consensus that molecular distinctions within and between individual tumours is an important factor determining clinical outcomes. Consequently, integrated approaches based on the combination of molecular profiling with traditional methods such as immunohistochemical phenotyping, karyotyping and/or non-quantitative methylation-specific PCR have emerged as a promising venue towards increasing the predictive value of diagnostics for malignant brain tumors. The high level of inter-and intra-tumoural molecular diversity in gliomas underscores the need of integrating high throughput molecular profiling and pharmacogenomics into a diagnostic paradigm for gliomas and raises the possibility that molecular-instructed personalized treatments may provide clinical benefit to patients with glioblastoma, particularly in the setting of post-treatment recurrence. Here we discuss potential prospects and challenges of patient-tailored diagnostics and personalized treatment strategies for recurrent glioblastomas.
\end{abstract}

Keywords: Glioblastoma; Glioblastoma recurrence; Glioma stem cells; Intratumoural heterogeneity; Molecular diagnostics; High throughput profiling; Personalised medicine

\section{Personalized Approach to Cancer Treatment}

Personalized cancer therapy is the most promising also most challenging area of research in modern oncology. Reflecting this fact, PubMed search by terms "personalized cancer therapy", "precision cancer treatment" or "individualized cancer therapy" yields 4619, 4982 and 3821 entries as of March 2017. The underlying concept is that molecular distinctions between individual tumours/tumour subtypes within the same cancer can provide important instructions for delineating most effective treatment schemes particularly for cancers poorly responding to conventional treatments. Consequently, developing patient-tailored individualised therapy (IT) has become recognized as a promising venue improving the accuracy of diagnosis and stratification of treatment schemes for patients with treatmentrefractory cancers [1-3]. A direct relationship between the level of genetic complexity and poor efficacy of therapeutic strategies based on the "one-treatment-for-all" principle can be seen in glioblastoma, a genetically complex cancer characterized by a high degree of molecular and cellular heterogeneity. The promise of high-throughput molecularbased diagnostics as a strategy for more accurate patient stratification and prognostic staging has been recognized as a matter of urgent priority for improving the efficacy of glioblastoma therapy [4,5]. In this article, we discuss the current state, perspectives and specific challenges in molecular characterization and development of personalized approaches for treatment-refractory glioblastoma.

\section{Glioblastoma: General Facts}

Glioblastoma is the most common, most aggressive and inevitably lethal brain tumor in adults [6]. A dismal prognosis in conjunction with limited treatment options place glioblastomas among most challenging human cancers today. Age and gender are the most significant risk factors. Glioblastoma is most frequently diagnosed at a later age (median age at diagnosis 64 years old), occur at slightly higher rates in men than women and have the highest rate of incidence in Caucasians [7-9]. Although a higher risk of developing gliobalstoma has been associated with radiation exposure and germ line mutations causing neurofibromatosis (NF1 and NF2), Li Fraumeni syndrome (TP53), melanoma-astrocytoma syndrome (CDKN2A), tuberous sclerosis (TSC1/TSC2), Turcot syndrome (mismatch repair genes) and 
Cowden syndrome (PTEN) [10-12] these factors account only for a very small number of patients, with most cases of glioblastoma being diagnosed sporadically. Although there is some evidence for an inverse association of glioma risk and allergic disease the reason for this association remains unclear [13]. Although a trend towards a slight increase in the incidence of brain tumors in developed countries has been reported $[14,15]$, this may be attributed to more readily available diagnostic imaging. However uncommon (3/100000 population per year) glioblastoma continue to be among the most cinically relevant human cancers posing a persistent clinical challenge due to lack of satisfactory treatments. The current standard of care for newly diagnosed glioblastoma (ndGB) involves maximum safe surgical resection followed by a combined therapy of external beam radiation and chemotherapy. The latest significant improvement in the treatment of glioblastoma dates back to 2005 when the alkylating agent temozolomide (TMZ) was added to radiation therapy (RT) as a part of standard therapy for glioblastoma [16]. Although the RT+TMZ regimen offers only a modest survival advantage compared to RT alone (median survival 14.6 vs. 12.2 months, respectively, 5-year survival $9.8 \%,[16]$ and is beneficial to only a subgroup of patients with glioblastomas lacking MGMT [17], TMZ remains a mainstay of chemotherapy for glioblastoma due to lack of better options. An inevitable recurrence after standard therapy is the ultimate cause of death from glioblastoma. Currently, there are no treatments for recurrent glioblastoma (recGB), with the lack of therapeutic options precluding any chance of disease control after recurrence $[5,18]$.

Glioblastomas recurr nearly inevitably after (or even during) standard therapy with tumour recurrence after the initial treatment being the ultimate cause of death [19]. For recGB, there is no standard treatment. Despite intensive efforts in the past decade to define therapeutically effective treatments for recGB none of the regimens tested yielded significant results $[5,19,20]$. Most of investigational therapies tested so far in recGBs have been based on a hypothesistesting approach aiming at addressing the following possibilities 1) Improving clinical outcomes through repeated application of the principle modules of standard unspecific therapy (surgery, RT or TMZ) in recGBs 2) Improving clinical outcomes through targetoriented treatments interfering with intrinsic (angiogenesis, invasion/ adhesion/ECM remodelling, proliferation) or extrinsic (tumourassociated immunosupression) mechanisms in recGB. So far, none of the studies testing various regimens of cytotoxic or targeted therapeis (alone or in combination with components of standard chemotherapy) in recGBs provided level one evidence for clinically significant improvement of survival in recGB [20]. Immunotherapeutic approaches based on autologous vaccination with dendritic cells have yielded some encouraging preliminary results in both ndGB and recGBs in a series of Phase I/II trials [21,22] but their confirmation in Phase III is still awaited. A comprehensive discussion of the outcomes of all completed trials on recGB has been covered in several recent reviews $[5,19,20]$.

The hypothesis-generating approach to delineate treatment strategies for glioblastomas has been generally less explored primarily due to limitations of conventional low-throughput methodologies [23]. Recent radical advances in high-throughput methodologies have opened the possibility of a systematic genome-wide profiling of tumour specimens in a standardized fashion [24]. Genome-wide profiling has been instrumental in providing a molecular explanation for the limited efficacy of the "same-treatment-for-all" approach in glioblastoma [25-27] and failure of certain types of treatments, particularly TMZ (and possibly other alkylating agents), to provide clinical benefit to patients with recGB [27]. Comparative analyses of patient-matched ndGBs and recGBs in the TCGA study revealed that $60 \%$ of recGBs acquire novel mutations whose pattern is consistent with a so-called hypermutator phenotype [27]. One of the most striking revelations was an association between new mutation patterns emerging in recGBs with the MGMT promoter methylation status. It was found that recGBs with the methylated status of MGMT promoter (MGMT $^{\mathrm{Me}+}$, silenced $M G M T$ gene, TMZ sensitive phenotype) show a distinct pattern of mutations different from that in $\mathrm{MGMT}^{\mathrm{Me}-}$ (activated MGMT gene, TMZ resistant phenotype) tumours. Consistent with the absence of MGMT function, a non-random pattern of CT substitutions occurring preponderantly at non-CpG sites in $\mathrm{MGMT}^{\mathrm{Me}+}$ recGBs was found [28]. Moreover, the spectrum of genes affected by newly acquired mutations in $\mathrm{MGMT}^{\mathrm{Me}+}$ recGBs suggests the emergence of a deficiency in global DNA repair driven by TMZ treatment. These findings reveal a previously unsuspected duality of the effects of TMZ (and possibly other DNA alkylating agents) in glioblastomas depending on the MGMT status: On one hand, TMZ exerts a stronger killing effect on $\mathrm{MGMT}^{\mathrm{Me}+}$ ndGBs but on the other hand, sets a strong selection pressure to lose DNA repair function in tumours that have initially been responsive to TMZ. As a consequence, increased accumulation of novel mutations is likely to lead to the development of secondary resistance to alkylating agents including TMZ in recurrent tumors with the $\mathrm{MGMT}^{\mathrm{Me}+}$ background due to DNA repair deficiency. An important implication from these findings is that cytotoxic treatments may proactively promote the emergence of new patterns of aberrantly expressed genes/oncopathways in recGBs with further molecular diversification depending on the molecular type of initial tumour. As a consequence of such treatment-driven and molecular type-dependent evolution, recGBs may considerably deviate in their molecular landscapes from precursor tumors. In agreement with this hypothesis, comparative profiling has revealed considerable differences between ndGBs and recGBs in mutational spectrum, frequency of genomic rearrangemens and gene expression patterns $[29,30]$. Notably, a considerable number of fusion transcripts identified in recGBs involve genes that have not previously been associated with gliomas suggesting that therapeutically actionable lesions in recGBs may differ from those identified in ndGBs. In the study by Riemer and co-authors more than $40 \%$ of recGBs showed genomic aberration patterns distinct from those in their ndGBs counterparts [30]. Based on the degree of (dis)similarity with the patterns of genomic aberrations characteristic of ndGBs, recGBs could be stratified into distinct molecular types termed "equal", "sequential" or "discrepant" [30]. The existence of genetically distinct subgroups across recGBs further indicates that recGBs are molecularly heterogeneous tumours that may evolve through clonal selection proactively enforced by cytotoxic treatments. Indeed, several recent studies have provided convincing evidence for molecular drifts occurring during glioblastoma progression after (or under) standard therapy. Xiu et al. have compared 12 pairs of patient-matched ndGBs and recGBs and found profound changes in the spectrum of biomarkers including MGMT methylation in $94 \%$ of samples analyzed [31]. Importantly, loss of MGMT methylation was found to be a more frequent event than acquisition in recGBs. Similarly, Kim et al. found a high discordance rate of $29 \%$ in the expression level of targetable lesions when comparing 10 pairs of patient-matched ndGBs and recGBs [32]. That molecular patterns characteristic of a particular subtype of ndGB undergo dynamic changes during recurrence [33] further supports the general hypothesis that recGBs are molecularly distinct from ndGBs and that independent assessment of biomarkers in recGBs is a 
Citation: Kalasauskas D, Renovanz M, Bikar S, Buzdin A, Enam A, et al. (2017) Perspectives and Challenges in Molecular-Based Diagnostics and Personalized Treatment for Recurrent High-Grade Gliomas. J Carcinog Mutagen 8: 290. doi:10.4172/2157-2518.1000290

Page 3 of 9

prerequisite for developing effective treatments in the setting of glioblastoma recurrence.

\section{Molecular Stratification of Recurrent Glioblasoma: Challenges and Possible Solutions}

There is a growing realization of the need for molecular classification as a pre-requisite to improve the diagnostic accuracy and develop treatment stratification schemes for recGB [4]. Highthroughput profiling has been instrumental in identifying four major molecular subtypes of glioblastoma termed classic, pro-neural, proliferative and mesenchymal $[26,34]$. The four subtypes described by Phillips et al. have now been widely accepted in neurooncology research as the standard for molecular grading of different types of glioma [35-41]. The past decade has been marked by an explosion of exploratory investigations that have applied high-throughput omics to characterizing (epi)genome, transcriptome and metabolome in ndGBs [42-44]. For recGBs, high-throughput profiling data are considerably scarcer [25,31-33]. One of the constraining factors is the limited availability of recGB tissue samples due to the fact that only around $25 \%$ of patients are considered for open surgery after recurrence [20] and that diagnostic biopsy is not routinely justified for recGBs due to lack of effective treatment options that can be derived from such a biopsy. An even greater constraint is posed by difficulties in obtaining patient-matched metachronous tumour samples in order to identify true changes associated with glioblastoma progression independent of inter-patient molecular heterogeneity. Another common limitation of existing studies is that profiling was mostly done using a single tumour sample. Such an approach relies upon the assumption that a single tumour sample is representative for the whole tumour. Glioblastomas are notorious for their high degree of intratumoural heterogeneity manifest in spatial and temporal pattern of molecular, phenotypic and functional diversity (Figure 1).

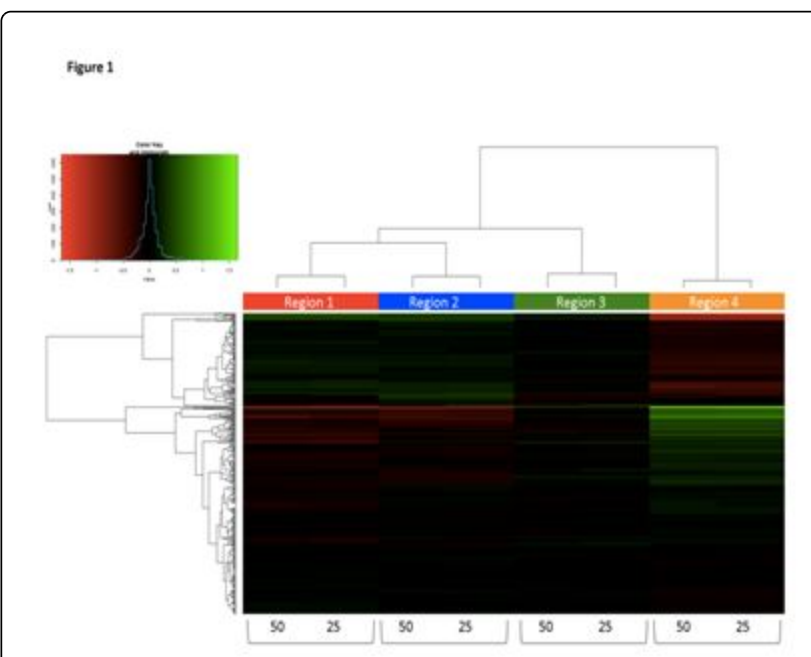

Figure 1: Heat map showing gene expression patterns identified in four regions from the same tumour.

Four samples resected at surgery from different regions of recurrent glioblastoma (WHO) were analyzed by RNA sequencing using the NextSeq 500 system from Illumina. Each sample was sequenced at $25 \mathrm{M}$ and $50 \mathrm{M}$ sequencing depth with $2 \times 150 \mathrm{bp}$ read length.
Recent studies [44,45] and our own research (Figure 1) reveal significant variations in the patterns of genomic alterations and gene expression between multiple samples derived from the same tumor. Intriguingly, Sottoriva et al. showed co-existence of different molecular patterns within the same tumor that were previously thought to be associated with clinically distinct subtypes of glioblastoma [26,34]. The underlying interconnected mechanisms involved in the emergence of intratumoural heterogeneity in glioblastoma involve genetic diversification and clonal expansion driven by inherent genomic instability [46], dynamic interconversions between distinct cell states and molecular patterns [47,48] and, in the setting of tumour recurrence, treatment-dependent molecular adaptation as discussed above.

The phenomenon of intratumoural heterogeneity has important implications for the interpretation of molecular data obtained from a single tumour sample and argues for the need to analyse multiple samples from the same tumour in order to minimize the risk of sampling bias [49]. While the association between intratumoural heterogeneity and development of treatment resistance has been widely recognized there are several challenging issues specific to glioblastomas. One is that implementing a multisampling approach to recGBs is not a trivial task in a clinical setting. For recurrent glioblastoma re-operation is considered justified under the premise that a number of criteria are met. These include the feasibility of gross total resection, likelihood of palliation of neurological deficits after surgery, low risk of developing new neurological deficit due to surgery, no contraindication to surgery due to the patient's general physical condition. However, the majority of recGBs are not suitable for resection at progression because of their invasion into eloquent brain causing neurological deterioration, spread through the ventricular system or the CSF pathways, and multifocal pattern [50]. One possible way to compensate for these limitations may be a biopsy-based approach to collecting tumour tissue samples. It has recently been shown that biopsy samples of 5-10 $\mathrm{mm}^{3}$ are well suited for performing high-throughput multiparameter profiling (genomic aberrations and gene expression patterns) in glioblastomas [44]. Using a multisampling approach Parker and co-authors showed that nearly half of the tumours in a set of 14 ndGBs and recGBs had mixed patterns of gene expression allocated to different regions spatially separated within the same tumor. Although biopsy samples were obtained during surgery in this study, the finding that $5-10 \mathrm{~mm}^{3}$ biopsy sample is enough for high-throughput profiling raises the possibility that a stereotactic needle biopsy used sometime for conventional diagnostics of brain tumours may be a valuable asset in high-throughput profiling for molecular diagnostics.

Currently, routine use of stereotactic needle biopsy for conventional diagnostics of recurrent glioblastoma is limited primarily due to the absence of treatment options. Although it carries a low surgical risk recommendation of stereotactic biopsy for recurrent glioblastoma is justified only if the diagnostic information is required for treatment decisions, for example delineating progression from treatmentassociated pseudoprogression. However, in the light of emerging data that high-throughput profiling may lead to the identification of therapeutically targetable lesions in recGB testing the feasibility of stereotactic biopsy as an approach to implementing genome-wide profiling for patient stratification in recurrent tumors is worth pursuing. In order to test this contention we propose a workflow for multisampling of glioblastoma tissue using a needle biopsy in conjunction with image-guided navigation (Figure 2). 


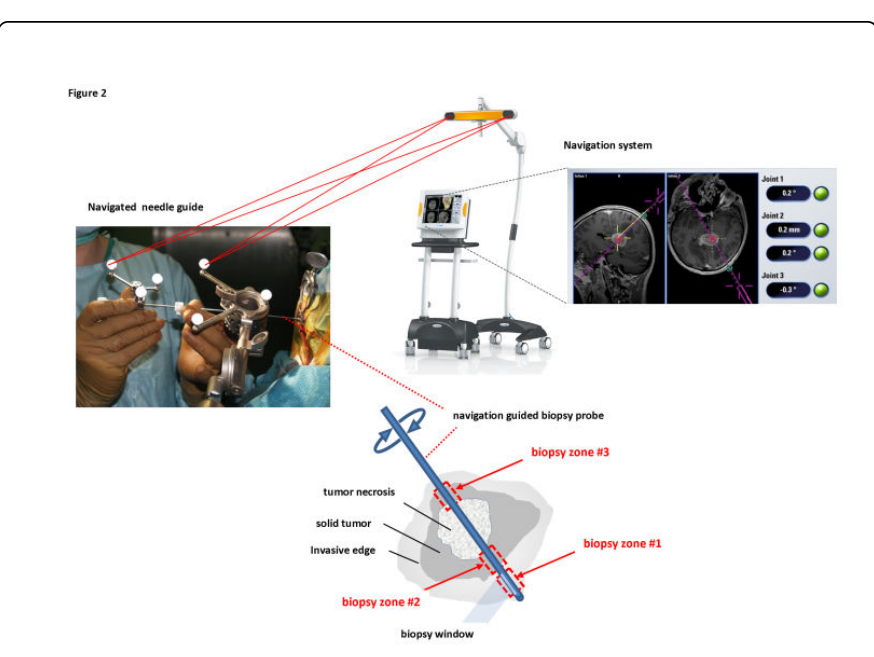

Figure 2: Multisampling of glioblastoma tissue using navigation guided needle biopsy.

A standard brain biopsy needle was registered to an optical tracking navigation device and guided by mechanical guidance unit (top, left). The trajectory and targets of the biopsy needle is pre-planned using preoperative MRI and the virtual needle position can be tracked in real time by the navigation system (top, right). The trajectory of the biopsy needle is planned to allow multiple biopsies within different biopsy zones, avoiding areas such as tumour necrosis or hemorrhage. Within the biopsy zones multiple biopsies (typically 1-4) may be taken by rotating the biopsy needle to position the biopsy window to different biopsy sites. The volume of biopsy samples is $4-8 \mathrm{~mm}^{3}$.

Such an approach enables to plan biopsy needle trajectories and perform tissue sampling from morphologically different regions of the tumour using a minimum of trajectories (preferentially a single trajectory). Early results are encouraging and support the idea that needle biopsy-based multisampling is a feasible and safe approach for implementing the concept of molecular-based diagnostics and treatment design in the majority of patients with recurrent glioblastomas including cases of non-resectable recGBs (as well as non-resectable ndGBs) and patients in poor neurological condition that carry the worst prognosis.

While the role of intratumoural molecular heterogeneity in therapy resistance of glioblastoma has been a focus of current research the question remains of why some glioblastomas show fairly homogeneous profiles of gene expression across different regions [44]. Is it a reflection of a lesser degree of molecular heterogeneity in a subset of glioblastomas or ambiquity, which is inevitably associated with analyses of whole tumour tissue samples?

The remaining conceptual question is what criteria should be used to stratify distinct signatures identified within the same tumour with respect to their prognostic value? The key to its solution is to elucidate the cellular and molecular mechanisms underlying glioblastoma progression especially during recurrence. In this regard, the impact of so-called glioma stem cells (GSCs) in treatment resistance and recurrence of glioblastomas has emerged as the primary focus of research. The current paradigm of gliomagenesis revolves around GSCs, which have been recognized as the most clinically relevant type of glioma cells that are primarily responsible for the maintenance of tumour growth [51]. Owing to their unique biological properties such as unlimited self-renewal propensity, infinite proliferative capacity, phenotypic and functional plasticity, and superior ability to survive cytotoxic insults that are lethal for non-stem gioma cells, GSCs have emerged as a key factor in therapy resistance and, consequently, a prime candidate for therapeutic targeting in GBs [52-56]. Delineating the molecular mechanisms underlying the GSCs ability to survive cytotoxic treatments and drive post-treatment growth of malignant gliomas has been recognized as the matter of prime importance to improve current therapeutic strategies. Dissecting the role of GSCs through whole-tissue analyses faces a number of methodological challenges mainly due to their rarity and non-homogeneous distribution within the tumour [57]. Furthermore, the relative number of GSCs undergoes dynamic changes during tumour progression [58,59]; last not least there is persistent uncertainty about GSCs markers $[56,60]$. A plethora of putative markers of GSC has been implicated in the past decade but their prognostic value remains uncertain. Adding further complexity, GSCs comprise diverse cell types differing in their phenotypic traits [60]. Despite the persistent uncertainty on GSC markers GSC can be selectively isolated based on the ability to propagate in vitro under selective cuture condition and be identified by distinct biological properties related to stemmness. These include the propensity for extended self-renewal, multipotentiality, ability to induce tumours and recapitulate morphological and molecular features of GBs in xenograft models [61]. Although it is generally accepted that GSCs are the closest from existing cell-based models of glioblastoma, there is no consensus in the literature on this point. Several studies have addressed the extent of resemblance between GSCs and tumour of their origin at the molecular level. It was shown that GSCs differ significantly in their gene expression and epigenetic signature from tumours of their origin, with GSCs isolated from ndGBs classified as mesenchymal (most aggressive) phenotype showing a proneural signature associated with less aggressive phenotype [47]. Conversely, it has also been shown that gene expression signatures closely match between GSCs and their tumours of origin [62]. Moreover, gene expression signatures characteristic of distinct glioblastoma subtypes were found to be more profound in GSCs than in their tumours of origin, likely reflecting the impact of intratumoural heterogeneity in tumour samples [62]. The reason for these discrepant findings remains unclear but may in part be attributable to heterogeneity of GSC types and intratumoural regional variations in the composition of GSC types [63]. By using single cell cloning approach Soeda and co-authors have recently shown that different types of molecularly distinct GSCs varying in the degree of tumorigenicity and drug sensitivities co-exist within the same tumour [63]. The emerging consensus is that intratumoural heterogeneity notorious in GBs is primarily contributed by GSCs owing to their inherent plasticity and capability to interconvert between different states [48]. The consideration of GSCs plasticity is especially important in the context of treatment-driven molecular drifts during glioblastoma recurrence. Several studies have shown that cytotoxic treatments such as radiation exposure induce transversion from a less aggressive to a more aggressive phenotype in GSCs [62]. A similar trend was revealed in the study by Huang et al. who used a different approach based on the comparison of GSCs isolated from patientmatched ndGB and recGB [64]. This study showed that GSCs isolated from recGB have a more aggressive phenotype than their precursor GSCs from ndGB [64]. Notably, cytogenetic analyses showed little difference in the patterns of genomic alterations between the two GSC populations further reinforcing the notion that global re-programming of gene expression provoked in GSCs by cytotoxic treatments may 
occur in the absence of global changes in the patterns of genomic alterations. Adding a further level of complexity, cytotoxic treatments can induce the conversion of glioma cells from non-stem to the GSC phenotype, representing yet another mechanism of therapy resistance [65]. Altogether, these findings strongly support the paradigm of glioblastoma recurrence driven by GSCs and demonstrate that GSC faithfully recapitulate key aspects associated with glioblastoma progression, namely acquisition of treatment resistant phenotype and treatment-induced molecular drifts. Another advantage of GSC models is that they provide the possibility to delineate molecular consequences of defined regimens of individual treatments/treatment combinations taking into account the temporal frames and dynamics of treatment-induced changes in GSCs. In contrast, whole tissue analyses do not allow dissecting the impact of individual treatments/ treatment regimens on different cell types due to clinical necessity of the concomitant use of radiation and chemotherapy. GSC-based models provide such an opportunity. Figure 3 illustrates an experimental strategy established in our group to delineate the molecular and biological consequences of different types of cytotoxic treatments in different types of GSCs.

Such an approach has several advantages compared to whole tissue analyses. First, the impacts of individual treatments/treatment regimens can be distinguished from mixed effects of combined treatment regimens. Second, the association between particular molecular subtypes of GSCs and therapy response can be delineated. Third, the temporal dynamics of treatment-induced molecular changes can be characterized. The latter aspect is directly related to the issue of optimal timing of a treatment switch to catch up with adaptive molecular changes induced by initial therapy.

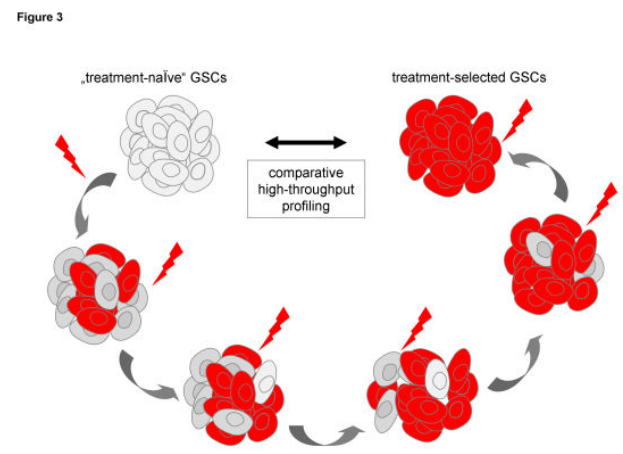

Figure 3: Experimental approach to identifying treatment-specific changes induced in GSC. To obtain treatment-resistant GSCs, treatment-naïve GSCs isolated are subjected to defined regimens of clinically relevant treatments and expanded in vitro. Isogenic pairs of treatment-naïve and treatment-selected populations can then be compared directly and in parallel on the cellular, molecular and tissue levels.

\section{Personalized Medicine Paradigm for the Treatment of Brain Tumours: Framework, Costs and Feasibility}

The three pillars of the personalized medicine paradigm are 1) highthroughput molecular profiling of individual tumors, 2) bioinformatics analyses of molecular readouts and 3) coordinated action of clinical oncologists to translate bioinformatics predictions into clinical practice by designing personalized treatments adapted to individual risk factors and patients' specific characteristics. Consequently, the successful implementation of this paradigm requires an interdisciplinary network to ensure a fast turnaround time from diagnosis to individualized treatments. Recent advances in molecular biotechnology and bioinformatics now make this a realistic task. Next Generation Sequencing (NGS, also known as high-throughput sequencing) provides standardized means of identifying genomic alterations, aberrant transcripts as well as undefined genomic regions (promoters, exons, non-coding RNAs and their variants, repetitive sequences, pseudogenes) and epigenetic patterns [66] with an unprecendented depth of informational output. Whole genome coverage, quantitative readouts, lesser impact of inter-performer variability due to the high level of standardized procedures and increasing affordability make NGS-based profiling an emerging standard methodology for molecular-based diagnostics.

Bioinformatics analytic tools are central in the application of "big data" in genomics and in translation of gene expression to clinical practice. Thus far, there are two major approaches, i.e. those based on the mutation analysis and those based on gene expression profiling. Most of existing predictor modes profile only a limited number of biomarkers, cover only a minor fraction of target drugs, and are limited to a particular type of cancer. It is clear now that more universal methods are required to rank a maximum number of existing drugs. Conveying the NGS gene-level data to the cellular signalling pathway level is key to predict functional consequences from transcriptomic profiling. However, most of the models cannot use data from multiple sequencing platforms, cannot efficiently handle the high-throughput quantification of pathway activation scores for individual biological samples required for clinical use and cannot meet the time frame needed for treatment of glioblastomas [67]. A method termed Oncofinder was recently developed for predicting target drug efficacy based on a patient's cancer-specific pattern of signalling pathway activation, particularly for pathways including molecular targets of respective drugs [68]. The enclosed algorithm operates with the so-called Pathway Activation Strength value, which is a qualitative characteristic of pathway activity in a cancer sample. This approach was used to identify molecular features accounting for the tumor response to target drugs in cell-based models [69] and patients [70]. In glioblastoma, 59 abnormally regulated intracellular signaling pathways could be revealed by Oncofinder [71]. Based on this knowledge patient/patient group specific response to various anticancer target drugs expressed by a value termed "drug score" can be predicted [68]. Drug scores for glioblastoma patients varied greatly and showed very strong individual patterns. Many tyrosine kinase inhibitors and monoclonal antibodies, which have never been used for glioblastoma treatment, showed high drug scores in individual cases (own unpublished data). This suggests that a personalized mode of treatment stratification for glioblastoma patients will be more effective than the current "one-treatment-for-all" treatment scheme.

Surgical and neurooncological treatment of brain tumors is costly and for malignant CNS tumors of limited effectiveness. Whereas the gain in overall survival time and quality of life for glioblastoma patients has made some progress, the funding spent per treatment of an average glioblastoma patients covering expenses on radiographic and histological diagnostics, surgical treatment, radiation treatment, neurooncological management, pharmaceuticals, and palliative care has been growing constantly. In Europe, a significant increase in costs 
associated with treatment of malignant gliomas has been primarily due to addition of TMZ as concomitant and adjuvant treatment, now administered well beyond six cycles originally evaluated in the pivotal EORTC trail with some extended protocols continuing TMZ until disease recurrence. This has increased the costs over radiation therapy alone significantly [72]. In Germany for example, now costs covered by health insurance companies for TMZ alone are on the order of a hundred million euros. In the USA treatment costs for concomitant radiation TMZ chemotherapy in the first year after surgery amounted to total healthcare costs of 184.000 \$ per glioma patient compared to $\$ 88,827$ for patients without adjuvant therapy [73]. The incremental cost-effectiveness ratios (ICERs) for cost per gained life-year corresponds to $€ 37.361$ [74]. The global costs associated with treatment of malignant gliomas in 2013 reached \$301 million and projected to double in the next decade. In the face of growing cancer burden and global economic slowdown, the need in new effective treatments that would be affordable for the majority of cancer patients is pressing as never before. Only a few years ago, the relatively high costs and long runtime have limited the utilization of high-through profiling in clinical diagnostics. Currently the situation is rapidly changing due to the rapid drop of NGS costs in recent years and broader availability of this methodology through the commercial biotechnology sector.

We have recently pioneered a workflow integrating specific expertise in clinical neurooncology, high-throughput profiling of gene expression and bioinformatics analysis for pathway activation and treatment prediction in glioblastomas (www.omicsglioma.com). Tissue specimens from surgical resections or stereotactic biopsies of malignant brain tumours using a multisampling approach are snap frozen in liquid nitrogen and stored at $-80^{\circ} \mathrm{C}$.

Specimens are divided for the neuropathology evaluation and highthroughput profiling by RNA sequencing. Extracted RNA with a RIN value of $>7$ is then used for the preparation of stranded RNA libraries. After the quality control (QC) of the sequencing libraries RNAsequencing is performed using the NextSeq 500 system from IlluminaTM at a sequencing depth of $25-75 \mathrm{M}$ reads with $2 \times 150 \mathrm{bp}$ read length.

After bioinformatics analysis with TopHat 2 [75], Cufflinks 2 [76], Isaak Variant caller [77] and TopHat-Fusion [78] the data are further analyzed using the OncoFinder algorithm. Based on a hierarchical ranking of aberrantly functioning genes and cellullar pathways anticancer agents with the closest match to the molecular profile of individual patients can be identified and ranked based on a score value assigned by the Oncofinder software.

The generated list of drug candidates provides an instructive recommendation that can further be used by neurooncologist for evaluation of individual risk profiles and design of treatment plans. Such an integrative approach based on task segmentation between academic medical centers and biotechnology and bioinformatics companies could provide means to accelerate the implementation of personalized diagnostics and its application in clinical practice.

Currently, personalized therapy is available only at academic medical centers in high-income countries. This is in large due to centralization of non-overlapping expertise (clinical care, availability of high-throughput methodology and bioinformatics specialized in multiparameter analysis of different datasets) required to implement personalized medicine in large academic centers.
Conceptually, a different type of framework based on task segmentation can be developed to provide practicing neurooncologists outside of highly specialized centers access to state-of-the-art treatment design almost everywhere (Figure 4).

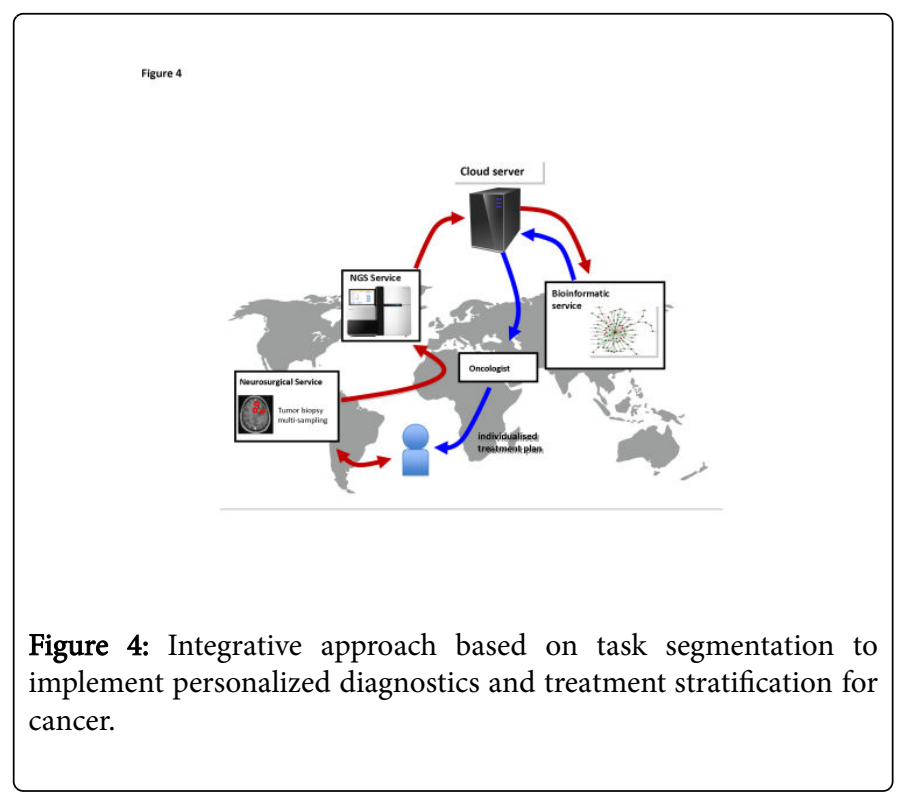

\section{Global Outlook}

According to WHO projections an unprecedented 50\% increase of annual cancer cases is expected within the next 2 decades [7,79]. A major contribution namely half of cancer death in 2008 occurs in low and middle income countries [79]. One of the reasons for the high rates of cancer deaths in low and middle income countries is insufficiency of cancer control measures and limited healthcare resources [79]. Considering the populations rapid growth and limited availability of adequate diagnostics and treatment modalities, establishing cost-effective neurooncology services in low and middle income countries where more than two thirds of all cancer deaths occur is a matter of urgent priority. A further persistent problem is the lack of accurate CNS tumor registries. For example, in Pakistan with its estimated population of more than 190 million in 2015, the epidemiological data and most of the reports on the incidence of brain tumors are based on single institution experiences [80]. Available data on molecular profiles in glioblastomas in South Asia suggests variations in the molecular profiles such as TP53 mutation, EGFR amplification, PTEN deletion and IDH1 mutation, when compared to the gliomas in North America [81,82]. The frequency of MGMT promoter methylation is also different from other populations across the world $[83,84]$. A study on TP53 in primary GBM has revealed new TP53 mutations in South Asian population, not reported in other populations [85]. Even though unproven, this may suggest distinct genomic, transcriptional, and epigenetic profiles in South Asian brain tumor patients. Consequently, different treatment strategies may be more effective in these populations. It is obvious that CNS tumors in low- and mid-income countries will have a significant socioeconomic impact with a foreseeable increase of resource burden on the healthcare sector in the future. This raises the question of strategies for future neurooncological services in developing countries. While it is obvious that low- and mid-income countries face a number of urgent problems requiring immediate solutions, the need in developing cost effective treatment strategies for brain tumors shares the same sense of 
urgency as in the rest of the world. In this regard, vast experience accumulated over more than 40 years of intense explorations of different therapeutic strategies since the declaration of War on Cancer [86] provides an extensive background that should be considered. In the context of the limited success of cytotoxic and targeted therapy approaches in glioblastoma, it is questionable whether neurooncological service in low- and mid-income countries should repeat the steps that have already proved limitedly effective in terms of outcome rates and costs. Does implementing the concept of "onetreatment-for-all" therapies at the edge of cost-effectiveness even in high-income countries make sense? The per capita income in Pakistan for example is $\$ 1513$ [87] and almost all the cost for medical treatment and care is born by the patient directly; only a few in the upper income carry third-party payer health insurance. Given the high costs and limited success of conventional concomitant RT/TMZ chemotherapy for glioblastoma it seems questionable whether further investments into such strategies and development of the required infrastructure especially for low- and middle-income countries is a good investment in the future.

On the other hand, de novo establishment of neurooncology services should consider the recent paradigm shifts necessitating a change in previous views on the glioma biology and, consequently, treatment concepts. The major question is whether it is a realizable task to set up the platform for molecular profiling and personalized diagnostic in a mid-income country? We argue that it is not only possible but absolutely necessary, to prevent a foreseeable loss in time, efforts and funds associated with establishing standard therapeutic regimens that have already proved to have low clinical efficacy and limited cost-effectiveness.

The explosive development of the biotechnology industry in recent years provides the possibility of rapid turnaround in obtaining readouts from tumour specimens. With improvement of highthroughput sequencing technology and increasing availability of these platforms the costs per biopsy will continue to decrease. Currently, with $\$ 1000$ per analysis the costs are approaching those of conventional diagnostics for malignant gliomas. Further, NGS profiling of the transcriptome inherently includes expression levels of diagnostic molecular markers including the transcripts of the MGMT gene, IDH1 and other markers currently screened for in routine diagnostics of brain tumors. This in the future may replace some of the conventional histology-based diagnostic procedures. The global shift from the geographic site-associated towards a web-based working mode interaction provide the possibility to build molecularly-guided individualized therapy practically at any site on the planet with the only requirement being accessibility to the internet and shipping service. Clearly, while all technical processes can be out sourced to specialized facilities located anywhere, onsite presence of interdisciplinary oncological teams familiar with the specific pharmacological characteristics of anti-cancer agents and specific risk profiles in a given patient will be required. Especially considering differences in the spectrum of anti-cancer agents available (and affordable) in different countries. In the context of South Asia, the availability of specific expertise in the field of natural products is of particular relevance. Traditionally oncologists in South Asia use natural products in cancer therapy. In fact, natural products or natural product-derived molecules constitute a major part of the FDA approved anti-cancer agents with Taxanes, Vinca alkaloids, podopyllotoxins and camptothecin being only some of the examples. Considering that molecular targets for many natural products and their derivatives are known, molecular profiling offers the possibility for molecular-based prediction of potentially effective natural products using the drug-repositioning approach [88]. On the experimental level pharmaceutical databases can be screened to identify compounds known to interfere with these pathways to include such candidates into the bioinformatics drug scoring and specific prediction models for gliomas.

\section{Conclusion}

Inevitable recurrence after standard cytotoxic therapy is a major challenge in the treatment of clinical management of glioblastomas. The need for therapies that would be effective against recurrent glioblastoma remains an urgent matter since decades. Recent advances in high-throughput profiling promoted molecular understanding of glioblastoma recurrence and led to the emergence of the current consensus that recurrent glioblastomas are molecularly distinct from their precursor tumours. An immediate implication is that therapeutically targetable lesions in recGBs may be distinct from those identified in ndGBs. A molecular classification of recurrent glioblastomas is prerequisite to the identification of molecular biomarkers and potential targets in these tumours. Glioma stem cells have emerged as a key determinant of intratumoural heterogeneity in both newly diagnosed and recurrent glioblastoma. In addition to inherent mechanisms underlying intratumoural heterogeneity treatment-driven selection is an important determining factor of the molecular diversification of recurrent glioblastoma. The implementation of personalized approach to the treatment of glioblastomas is a matter of global importance. Despite existing challenges the implementation of personalized approach to the treatment of glioblastoma in developing countries is a feasible task, which may be realized through the international, interdisciplinary and mutually beneficial networking between clinicians, academia and biotechnology sectors.

\section{Aknowledgement}

This work was in part supported by the German Federal Ministry of Education and Research (BMBF) and administered by Projektträger Julich (PTJ) under Work programme EUROTRANS-BIO (Call ETB-10), grant 031B0145B to EK, AG, SK, SB and AB.

\section{References}

1. Schilsky RL (2010) Personalized medicine in oncology: the future is now. Nature reviews Drug discovery 9: 363-366.

2. Wistuba II, Gelovani JG, Jacoby JJ, Davis SE, Herbst RS (2011) Methodological and practical challenges for personalized cancer therapies. Nature reviews Clinical oncology 8: 135-141.

3. Simon R, Roychowdhury S (2013) Implementing personalized cancer genomics in clinical trials. Nature reviews Drug discovery 12: 358-369.

4. Campos B, Olsen LR, Urup T, Poulsen H (2016) A comprehensive profile of recurrent glioblastoma. Oncogene 35: 5819-5825.

5. Mallick S, Benson R, Hakim A, Rath GK (2016) Management of glioblastoma after recurrence: A changing paradigm. J Egypt Natl Canc Inst 28: 199-210.

6. Louis DN, Perry A, Reifenberger G, von Deimling A, Figarella-Branger D, et al. (2016) The 2016 World Health Organization classification of tumors of the central nervous system: a summary. Acta neuropathologica 131: 803-820.

7. Stewart BW, Wild C (2014) World cancer report 2014: World Health Organization. 
8. Thakkar JP, Dolecek TA, Horbinski C, Ostrom QT, Lightner DD, et al (2014) Epidemiologic and molecular prognostic review of glioblastoma. Cancer Epidemiology and Prevention Biomarkers 23: 1985-1996.

9. Ostrom QT, Bauchet L, Davis FG, Deltour I, Fisher JL, et al. (2014) The epidemiology of glioma in adults: a "state of the science" review. Neurooncology 7: nou087.

10. Schwartzbaum JA, Fisher JL, Aldape KD, Wrensch M (2006) Epidemiology and molecular pathology of glioma. Nature clinical practice Neurology 2: 494-503.

11. Ohgaki H (2009) Epidemiology of brain tumors. Cancer Epidemiology 472: 323-342.

12. Ricard D, Idbaih A, Ducray F, Lahutte M, Hoang-Xuan K, et al. (2012) Primary brain tumours in adults. The Lancet 379: 1984-1996.

13. Schoemaker MJ, Swerdlow AJ, Hepworth SJ, McKinney PA, Van Tongeren $\mathrm{M}$, et al. (2006) History of allergies and risk of glioma in adults. International journal of cancer 119: 2165-2172.

14. Khurana VG, Jain S, Smee R, Cook R, Dobes M, et al. (2011) Increasing incidence of glioblastoma multiforme and meningioma, and decreasing incidence of Schwannoma (2000-2008): Findings of a multicenter Australian study. Surgery Neurology International 2: 176

15. Hardell L, Carlberg M, Hansson MK (2011) Pooled analysis of casecontrol studies on malignant brain tumours and the use of mobile and cordless phones including living and deceased subjects. International Journal of Oncology 38: 1465.

16. Stupp R, Hegi ME, Mason WP, van den Bent MJ, Taphoorn MJ, et al. (2009) Effects of radiotherapy with concomitant and adjuvant temozolomide versus radiotherapy alone on survival in glioblastoma in a randomised phase III study: 5-year analysis of the EORTC-NCIC trial. The lancet oncology 10: 459-466.

17. Hegi ME, Liu L, Herman JG, Stupp R, Wick W, et al. (2008) Correlation of O6-methylguanine methyltransferase (MGMT) promoter methylation with clinical outcomes in glioblastoma and clinical strategies to modulate MGMT activity. Journal of clinical oncology 26: 4189-4199.

18. Weller M, Cloughesy T, Perry JR, Wick W (2013) Standards of care for treatment of recurrent glioblastoma-are we there yet? Neuro-oncology 15: 4-27.

19. Weller M, Wick W, Aldape K, Brada M, Berger M, et al. (2014) Glioma. Nature reviews Disease primers 1: 15017.

20. Gallego O (2015) Nonsurgical treatment of recurrent glioblastoma Current oncology 22: e273.

21. Mohme M, Neidert MC, Regli L, Weller M, Martin R (2014) Immunological challenges for peptide-based immunotherapy in glioblastoma. Cancer treatment reviews 40: 248-258.

22. Chang CN, Huang YC, Yang DM, Kikuta K, Wei KJ, et al. (2011) A phase I/II clinical trial investigating the adverse and therapeutic effects of a postoperative autologous dendritic cell tumor vaccine in patients with malignant glioma. Journal of clinical neuroscience 18: 1048-1054.

23. Biesecker LG (2013) Hypothesis-generating research and predictive medicine. Genome research 23: 1051-1053.

24. Mrugala MM (2013) Advances and challenges in the treatment of glioblastoma: a clinician's perspective. Discovery medicine 15: 221-230.

25. Chen Y, Wu JJ, Lin XB, Bao Y, Chen ZH, et al. (2015) Differential lncRNA expression profiles in recurrent gliomas compared with primary gliomas identified by microarray analysis. International journal of clinical and experimental medicine 8: 5033.

26. Phillips HS, Kharbanda S, Chen R, Forrest WF, Soriano RH, et al. (2006) Molecular subclasses of high-grade glioma predict prognosis, delineate a pattern of disease progression, and resemble stages in neurogenesis. Cancer cell 9: 157-173.

27. McLendon R, Friedman A, Bigner D, Van Meir EG, Brat DJ, et al. (2008) Comprehensive genomic characterization defines human glioblastoma genes and core pathways. Nature 455: 1061-1068.

28. Esteller M, Herman JG (2004) Generating mutations but providing chemosensitivity: the role of O6-methylguanine DNA methyltransferase in human cancer. Oncogene 23: 1-8.
29. Bao ZS, Chen HM, Yang MY, Zhang CB, Yu K, et al. (2014) RNA-seq of 272 gliomas revealed a novel, recurrent PTPRZ1-MET fusion transcript in secondary glioblastomas. Genome research 24: 1765-1773.

30. Riehmer V, Gietzelt J, Beyer U, Hentschel B, Westphal M, et al. (2014) Genomic profiling reveals distinctive molecular relapse patterns in IDH1/2 wild-type glioblastoma. Genes, Chromosomes and Cancer 53: 589-605.

31. Xiu J, Piccioni D, Juarez T, Pingle SC, Hu J, et al. (2016) Multi-platform molecular profiling of a large cohort of glioblastomas reveals potential therapeutic strategies. Oncotarget 7: 21556.

32. Kim L, Xiu J, Judy K, Evans J, Andrews D, et al. (2016) MPTH-55. Results of molecular profiling for recurrent malignant gliomas reveal significant changes in biomarkers compared to motly treatment naïve tumors that could impact treatment decision. Neuro-oncology 18: vil17-vil18.

33. Kwon SM, Kang SH, Park CK, Jung S, Park ES, et al. (2015) Recurrent glioblastomas reveal molecular subtypes associated with mechanistic implications of drug-resistance. PLoS One 10: e0140528.

34. Verhaak RG, Hoadley KA, Purdom E, Wang V, Qi Y, et al. (2010) Integrated Genomic Analysis Identifies Clinically Relevant Subtypes of Glioblastoma Characterized by Abnormalities in PDGFRA, IDH1, EGFR, and NF1. Cancer cell 17: 98-110.

35. Guan X, Vengoechea J, Zheng S, Sloan AE, Chen Y, et al. (2014) Molecular subtypes of glioblastoma are relevant to lower grade glioma. PLoS One 9: e91216.

36. Gerber NK, Goenka A, Turcan S, Reyngold M, Makarov V, et al. (2014) Transcriptional diversity of long-term glioblastoma survivors. Neurooncology 16: 1186-1195.

37. Reifenberger G, Weber RG, Riehmer V, Kaulich K, Willscher E, et al (2014) Molecular characterization of long-term survivors of glioblastoma using genome-and transcriptome-wide profiling. International journal of cancer 135: 1822-1831.

38. Agnihotri S, Aldape KD, Zadeh G (2014) Isocitrate dehydrogenase status and molecular subclasses of glioma and glioblastoma. Neurosurgical focus 37: E13.

39. Purkait S, Mallick S, Sharma V, Kumar A, Pathak P, et al. (2016) A simplified approach for molecular classification of glioblastomas (GBMs): experience from a tertiary care center in India. Brain tumor pathology 33: 183-190.

40. Erdem-Eraslan L, Gravendeel LA, de Rooi J, Eilers PH, Idbaih A, et al. (2012) Intrinsic molecular subtypes of glioma are prognostic and predict benefit from adjuvant procarbazine, lomustine, and vincristine chemotherapy in combination with other prognostic factors in anaplastic oligodendroglial brain tumors: a report from EORTC study 26951. Journal of clinical oncology 31: 328-336.

41. Ceccarelli M, Barthel FP, Malta TM, Sabedot TS, Salama SR, et al. (2016) Molecular profiling reveals biologically discrete subsets and pathways of progression in diffuse glioma. Cell 164: 550-563.

42. Li QJ, Cai JQ, Liu CY (2016) Evolving molecular genetics of glioblastoma. Chinese medical journal 129: 464.

43. Alentorn A, Duran-Peña A, Pingle SC, Piccioni DE, Idbaih A, et al (2015) Molecular profiling of gliomas: potential therapeutic implications. Expert review of anticancer therapy 15: 955-962.

44. Parker NR, Hudson AL, Khong P, Parkinson JF, Dwight T, et al. (2016) Intratumoral heterogeneity identified at the epigenetic, genetic and transcriptional level in glioblastoma. Scientific reports 6: 23.

45. Sottoriva A, Spiteri I, Piccirillo SG, Touloumis A, Collins VP, et al. (2013) Intratumor heterogeneity in human glioblastoma reflects cancer evolutionary dynamics. Proceedings of the National Academy of Sciences 110: 4009-4014.

46. Andor N, Graham TA, Jansen M, Xia LC, Aktipis CA, et al. (2016). Pancancer analysis of the extent and consequences of intratumor heterogeneity. Nature medicine 22: 105-113.

47. Bhat KP, Balasubramaniyan V, Vaillant B, Ezhilarasan R, Hummelink K, et al. (2013) Mesenchymal differentiation mediated by NF- $\kappa B$ promotes radiation resistance in glioblastoma. Cancer cell 24: 331-346. 
Citation: Kalasauskas D, Renovanz M, Bikar S, Buzdin A, Enam A, et al. (2017) Perspectives and Challenges in Molecular-Based Diagnostics and Personalized Treatment for Recurrent High-Grade Gliomas. J Carcinog Mutagen 8: 290. doi:10.4172/2157-2518.1000290

Page 9 of 9

48. Safa AR, Saadatzadeh MR, Cohen-Gadol AA, Pollok KE, BijangiVishehsaraei K (2015) Glioblastoma stem cells (GSCs) epigenetic plasticity and interconversion between differentiated non-GSCs and GSCs. Genes \& Diseases 2: 152-163.

49. Renovanz M, Kim EL (2015) Intratumoral heterogeneity, its contribution to therapy resistance and methodological caveats to assessment. cancer 2015: 121.

50. Giese A, Bjerkvig R, Berens M, Westphal M (2003) Cost of migration: invasion of malignant gliomas and implications for treatment. Journal of clinical oncology 21: 1624-1636.

51. Codrici E, Enciu AM, Popescu ID, Mihai S, Tanase C (2016) Glioma stem cells and their microenvironments: providers of challenging therapeutic targets. Stem cells international 2016: 5728438.

52. Stopschinski BE, Beier CP, Beier D (2013) Glioblastoma cancer stem cells-from concept to clinical application. Cancer letters 338: 32-40.

53. Jackson M, Hassiotou F, Nowak A (2014) Glioblastoma stem-like cells: at the root of tumor recurrence and a therapeutic target. Carcinogenesis 36: 177-185.

54. Cheng L, Bao S, Rich JN (2010) Potential therapeutic implications of cancer stem cells in glioblastoma. Biochemical pharmacology 80: 654-665.

55. Huang Z, Cheng L, Guryanova OA, Wu Q, Bao S (2010) Cancer stem cells in glioblastoma-molecular signaling and therapeutic targeting. Protein \& cell 1: 638-655.

56. Lathia JD, Mack SC, Mulkearns-Hubert EE, Valentim CL, Rich JN (2015) Cancer stem cells in glioblastoma. Genes \& development 29: 1203-1217.

57. Schiffer D, Mellai M, Annovazzi L, Caldera V, Piazzi A, et al. (2014) Stem cell niches in glioblastoma: a neuropathological view. BioMed research international 2: 725921.

58. Natsume A, Ito M, Katsushima K, Ohka F, Hatanaka A, et al. (2013) Chromatin regulator PRC2 is a key regulator of epigenetic plasticity in glioblastoma. Cancer Research 73: 4559-4570.

59. Ivanov VN, Hei TK (2014) Radiation-induced glioblastoma signaling cascade regulates viability, apoptosis and differentiation of neural stem cells (NSC). Apoptosis 19: 1736-1754.

60. Bradshaw A, Wickremsekera A, Tan ST, Peng L, Davis PF, et al. (2016) Cancer stem cell hierarchy in glioblastoma multiforme. Frontiers in surgery 3: 48 .

61. Tirino V, Desiderio V, Paino F, De Rosa A, Papaccio F, et al. (2013) Cancer stem cells in solid tumors: an overview and new approaches for their isolation and characterization. The FASEB journal 27: 13-24.

62. Mao P, Joshi K, Li J, Kim SH (2013) Mesenchymal glioma stem cells are maintained by activated glycolytic metabolism involving aldehyde dehydrogenase 1A3. Proceedings of the National Academy of Sciences 110: 8644-8649.

63. Soeda A, Hara A, Kunisada T, Yoshimura Si, Iwama T, et al. (2015) The evidence of glioblastoma heterogeneity. Scientific reports 5: 7979.

64. Huang Q, Zhang QB, Dong J, Wu YY, Shen YT, et al. (2008) Glioma stem cells are more aggressive in recurrent tumors with malignant progression than in the primary tumor, and both can be maintained long-term in vitro. BMC cancer 8: 304.

65. Heddleston JM, Li Z, McLendon RE, Hjelmeland AB, Rich JN (2009) The hypoxic microenvironment maintains glioblastoma stem cells and promotes reprogramming towards a cancer stem cell phenotype. Cell Cycle 8: 3274-3284.

66. Hurd PJ (2010) The era of epigenetics. Briefings in functional genomics 9: 425-428.

67. Buzdin AA, Zhavoronkov AA, Korzinkin MB, Venkova LS, Zenin AA, et al. (2014) Oncofinder, a new method for the analysis of intracellular signaling pathway activation using transcriptomic data. Frontiers in genetics 5: 55 .

68. Artemov A, Aliper A, Korzinkin M, Lezhnina K, Jellen L, et al. (2015) A method for predicting target drug efficiency in cancer based on the analysis of signaling pathway activation. Oncotarget 6: 29347.
69. Venkova L, Aliper A, Suntsova M, Kholodenko R, Shepelin D, et al. (2015) Combinatorial high-throughput experimental and bioinformatic approach identifies molecular pathways linked with the sensitivity to anticancer target drugs. Oncotarget 6: 27227.

70. Zhu Q, Izumchenko E, Aliper AM, Makarev E, Paz K, et al. (2015) Pathway activation strength is a novel independent prognostic biomarker for cetuximab sensitivity in colorectal cancer patients. Human genome variation 2: 15009 .

71. Borisov NM, Terekhanova NV, Aliper AM, Venkova LS, Smirnov PY, et al. (2014) Signaling pathways activation profiles make better markers of cancer than expression of individual genes. Oncotarget 5: 10198-101205.

72. Wasserfallen JB, Ostermann S, Pica A, Mirimanoff RO, Leyvraz S, et al. (2014) Can we afford to add chemotherapy to radiotherapy for glioblastoma multiforme? Cost-identification analysis of concomitant and adjuvant treatment with temozolomide until patient death. Cancer 101: 2098-2105.

73. Ray S, Bonafede MM, Mohile NA (2014) Treatment Patterns, Survival, and Healthcare Costs of Patients with Malignant Gliomas in a Large US Commercially Insured Population. American health \& drug benefits 7 .

74. Lamers LM, Stupp R, van den Bent MJ, Al MJ, Gorlia T, et al. (2008) Cost-effectiveness of temozolomide for the treatment of newly diagnosed glioblastoma multiforme. Cancer 112: 1337-1344.

75. Kim D, Pertea G, Trapnell C, Pimentel H, Kelley R, et al. (2013) TopHat2: accurate alignment of transcriptomes in the presence of insertions, deletions and gene fusions. Genome biology 14: R36.

76. Trapnell C, Williams BA, Pertea G, Mortazavi A, Kwan G, et al. (2010) Transcript assembly and quantification by RNA-Seq reveals unannotated transcripts and isoform switching during cell differentiation. Nature biotechnology 28: 511-515.

77. Raczy C, Petrovski R, Saunders CT, Chorny I, Kruglyak S, et al. (2013) Isaac: ultra-fast whole-genome secondary analysis on Illumina sequencing platforms. Bioinformatics 29: 2041-2043.

78. Kim D, Salzberg SL (2011) TopHat-Fusion: an algorithm for discovery of novel fusion transcripts. Genome biology 12: R72.

79. International Network for Cancer Treatment and Research (2015) Cancer in Developing Countries.

80. Moore MA, Ariyaratne Y, Badar F, Bhurgri Y, Datta K, et al. (2010) Cancer epidemiology in South Asia-past, present and future. Asian Pac J Cancer Prev 11: 49-66.

81. Jha P, Suri V, Singh G, Jha P, Purkait S, et al. (2011) Characterization of molecular genetic alterations in GBMs highlights a distinctive molecular profile in young adults. Diagnostic Molecular Pathology 20: 225-232.

82. Jha P, Suri V, Sharma V, Singh G, Sharma MC, et al. (2011) IDH1 mutations in gliomas: first series from a tertiary care centre in India with comprehensive review of literature. Experimental and molecular pathology 91: 385-393.

83. Singh D, Chan JM, Zoppoli P, Niola F, Sullivan R, et al. (2012) Transforming fusions of FGFR and TACC genes in human glioblastoma. Science 337: 1231-1235.

84. Das P, Puri T, Jha P, Pathak P, Joshi N, et al. (2011) A clinicopathological and molecular analysis of glioblastoma multiforme with long-term survival. Journal of clinical neuroscience 18: 66 .

85. Phatak P, Selvi SK, Divya T, Hegde A, Hegde S (2002) Alterations in tumour suppressor gene $\mathrm{p} 53$ in human gliomas from Indian patients. Journal of biosciences 27:673-678.

86. National Cancer (1972) The National Cancer Act of 1971. Journal of the National Cancer Institute 48: 577-584.

87. Rana S (2016) Per capita income: A Pakistani now makes $\$ 1,513$ a year.

88. Sheridan H, Krenn L, Jiang R, Sutherland I, Ignatova S, et al. (2012) The potential of metabolic fingerprinting as a tool for the modernisation of TCM preparations. Journal of Ethnopharmacology 140: 482-491. 